\title{
The reduced distribution functions for charged particles with many-body interactions
}

\author{
T.Kahlbaum* \\ Institut für Plasma- und Lasertechnik, \\ Technische Fachhochschule Wildau, \\ Friedrich-Engels-Straße 63, Haus 8, D-15745 Wildau, Germany
}

Received August 14, 2000

We consider a multi-component system of charged particles that interact via a general many-body potential, and calculate the reduced distribution functions from the Bogoliubov-Born-Green-Kirkwood-Yvon hierarchy of equations in the form of a screened virial expansion through second order.

Key words: charged particles, distribution function, BBGKY hierarchy, virial expansion

PACS: $05.20 .-y$

\section{Introduction}

The calculation of the spatial distribution of particles in a given physical system with defined microscopic interactions belongs to the most fundamental problems in the statistical theory of the equilibrium properties of matter. If the particle species of the system and the global features of the relevant interaction potentials are known, one can derive the appropriate Bogoliubov-Born-Green-Kirkwood-Yvon (BBGKY) hierarchy as the basic set of equations for the distribution functions (see, e.g., [1]). Here, it is of particular interest to state whether these potentials are short-ranged, long-ranged, or even have components of both types, since as soon as long-range interaction is involved, the BBGKY hierarchy must be screened in a suitable way to insure convergence of the results.

The present paper deals with a multi-component system of classical particles that may differ in their mass, charge, and internal structure, and interact through a very general potential describing the two-body Coulomb forces, and arbitrary short-range forces between two and more particles as well. In the first part, we

*E-mail: torsten.kahlbaum@berlin.de 
fully specify the steps in the derivation of the BBGKY hierarchy for this system, starting from the definition of the reduced distribution function and proceeding to a decoupled set of differential equations for the coefficients in its virial expansion, where the adopted screening procedure was proposed by Schmitz $[2,3]$. The second part then presents details of getting analytic solutions of these equations for the first and the second-order virial coefficients which are given as multiple integrals over various combinations of the screened Coulomb potential and generalized Mayer cluster functions.

It should be mentioned that the exact determination of the virial expansion of the distribution function up to a certain order is only the starting-point in our whole programme to calculate the thermodynamic properties of non-ideal quantum plasma at low densities within the framework of the effective-potential approach [4,5], and a series of further papers on this subject will appear in the near future. For the sole purpose of obtaining the corresponding Helmholtz free energy, however, a formal treatment of the screened BBGKY hierarchy in terms of Mayer functions is quite sufficient, and hence we made no efforts to explicitly evaluate the integrals in the virial coefficients for a special choice of the short-range potentials as, e. g., hard-core interaction [6]. A brief account of the present results has been already published [7].

\section{The screened BBGKY hierarchy}

Let us examine a fluid mixture in the volume $V$ at temperature $T$ consisting of a total number of $N$ charged particles with spatial coordinates $\left\{\mathbf{r}_{i}\right\}(i=1, \ldots, N)$ whose species are denoted by either $a_{1}, a_{2}, \ldots$ or $a, b, \ldots$, so that $N=\sum_{a} N_{a}$, where $N_{a}$ is the number of particles of species $a$. In order to shorten our notation, we only retain all subscripts for the species but omit the coordinates which always means that, for any function, $\mathcal{F}_{a_{1} a_{2} \ldots} \equiv \mathcal{F}_{a_{1} a_{2} \ldots}\left(\mathbf{r}_{1}, \mathbf{r}_{2}, \ldots\right)$. The interaction between the $N$ particles is described by the general many-body potential $w_{a_{1} \ldots a_{N}}$ [8] made up of all two-, three- $, \ldots, N$-particle contributions $u_{a b}, u_{a b c}, \ldots, u_{a_{1} \ldots a_{N}}$ respectively,

$$
w_{a_{1} \ldots a_{N}}=\sum_{i<j=1}^{N} u_{a_{i} a_{j}}+\sum_{i<j<k=1}^{N} u_{a_{i} a_{j} a_{k}}+\cdots+u_{a_{1} \ldots a_{N}}
$$

This interacting multi-component system will be treated with the conventional methods of classical statistics, and the specific objective of our work is to relate the spatial distributions of the particles with the irreducible, or component, potentials $u_{a_{1} \ldots a_{\lambda}}(\lambda=2,3, \ldots, N)$ in equation $(1)$. We begin the calculation by making a random selection of $s$ particles from the system, $2 \leqslant s<N$, for which we define the reduced distribution function $f_{a_{1} \ldots a_{s}}$ as an integral over the exponential of the total, or direct, potential $w_{a_{1} \ldots a_{N}}$ with respect to the coordinates of the $N-s$ remaining particles,

$$
f_{a_{1} \ldots a_{s}}=\frac{V^{s}}{Q_{N}} \int \mathrm{d} \mathbf{r}_{s+1} \ldots \mathrm{d} \mathbf{r}_{N} \exp \left(-\beta w_{a_{1} \ldots a_{N}}\right)
$$


$\beta=1 / k_{\mathrm{B}} T$ is the inverse temperature, and the well-known configuration integral

$$
Q_{N}=\int \mathrm{d} \mathbf{r}_{1} \ldots \mathrm{d} \mathbf{r}_{N} \exp \left(-\beta w_{a_{1} \ldots a_{N}}\right)
$$

in the denominator of the prefactor in equation (2) guarantees the normalization of the distribution to $V^{s}, \int \mathrm{d} \mathbf{r}_{1} \ldots \mathrm{d} \mathbf{r}_{s} f_{a_{1} \ldots a_{s}}=V^{s}$, i. e., $f_{a_{1} \ldots a_{s}}$ is a dimensionless function.

In accordance with the separation of the $N$ particles into $s$ and $N-s$ particles, the total interaction potential, equation (1), can be decomposed into the contributions $w_{a_{1} \ldots a_{s}}$ from the $s$-particle system and $w_{a_{s+1} \ldots a_{N}}$ from the $(N-s)$-particle system, and the sum of contributions from the mutual interactions between these two systems,

$$
w_{a_{1} \ldots a_{N}}=w_{a_{1} \ldots a_{s}}+w_{a_{s+1} \ldots a_{N}}+\sum_{\alpha=1}^{N-s} w_{a_{1} \ldots a_{s}, a_{s+1} \ldots a_{N}}^{(\alpha)},
$$

where $w_{a_{1} \ldots a_{s}, a_{s+1} \ldots a_{N}}^{(\alpha)}$ is the composite potential for the interaction between all the $s$ particles and $\alpha$ particles from the $(N-s)$-particle system which starts with a $(1+\alpha)$-particle contribution and ends with an $(s+\alpha)$-particle contribution, $\alpha>0$,

$$
\begin{aligned}
w_{a_{1} \ldots a_{s}, a_{s}+1 \ldots a_{N}}^{(1)} & =\sum_{i=1}^{s} \sum_{\mu=s+1}^{N} u_{a_{i} a_{\mu}}+\sum_{i<j=1}^{s} \sum_{\mu=s+1}^{N} u_{a_{i} a_{j} a_{\mu}}+\cdots+\sum_{\mu=s+1}^{N} u_{a_{1} \ldots a_{s} a_{\mu}}, \\
w_{a_{1} \ldots a_{s}, a_{s+1} \ldots a_{N}}^{(2)} & =\sum_{i=1}^{s} \sum_{\mu<\nu=s+1}^{N} u_{a_{i} a_{\mu} a_{\nu}}+\cdots+\sum_{\mu<\nu=s+1}^{N} u_{a_{1} \ldots a_{s} a_{\mu} a_{\nu}}, \\
& \vdots \\
w_{a_{1} \ldots a_{s}, a_{s+1} \ldots a_{N}}^{(N-s)} & =\sum_{i=1}^{s} u_{a_{i} a_{s+1} \ldots a_{N}}+\sum_{i<j=1}^{s} u_{a_{i} a_{j} a_{s+1} \ldots a_{N}}+\cdots+u_{a_{1} \ldots a_{N}},
\end{aligned}
$$

or, in compact form,

$$
w_{a_{1} \ldots a_{s}, a_{s+1} \ldots a_{N}}^{(\alpha)}=\sum_{p=1}^{s} \sum_{\mu_{1}<\cdots<\mu_{p}=1}^{s} \sum_{\nu_{1}<\cdots<\nu_{\alpha}=s+1}^{N} u_{a_{\mu_{1} \ldots a_{\mu_{p}} a_{\nu_{1}} \ldots a_{\nu_{\alpha}}} .} .
$$

Note that the potentials for the separated systems, $w_{a_{1} \ldots a_{s}}$ and $w_{a_{s+1} \ldots a_{N}}$, are given by expressions like equation (1) but with sums running from 1 to $s$ and from $s+1$ to $N$ respectively, and one may include $\alpha=0$ by realizing that $w_{a_{1} \ldots a_{s}} \equiv w_{a_{1} \ldots a_{s}, a_{s+1} \ldots a_{N}}^{(0)}$.

Now we set out to derive an equation for the distribution function, equation (2), by taking the gradient $\nabla_{\mathbf{r}} \equiv \partial / \partial \mathbf{r}$ of $f_{a_{1} \ldots a_{s}}$ with respect to any coordinate as, e. g., $\mathbf{r}_{1}$ and using for $w_{a_{1} \ldots a_{N}}$ the above decomposition. The second term in equation (4), $w_{a_{s+1} \ldots a_{N}}$, does not contribute to this partial derivative, and with equation (8) we immediately find

$$
\begin{gathered}
\nabla_{\mathbf{r}_{1}} f_{a_{1} \ldots a_{s}}+f_{a_{1} \ldots a_{s}} \nabla_{\mathbf{r}_{1}} \beta w_{a_{1} \ldots a_{s}}+\sum_{p=1}^{s} \sum_{\mu_{1}<\cdots<\mu_{p}=1}^{s} \sum_{\alpha=1}^{N-s} \sum_{\nu_{1}<\cdots<\nu_{\alpha}=s+1}^{N} \\
\frac{1}{V^{\alpha}} \int \mathrm{d} \mathbf{r}_{\nu_{1}} \ldots \mathrm{d} \mathbf{r}_{\nu_{\alpha}} f_{a_{1} \ldots a_{s} a_{\nu_{1}} \ldots a_{\nu_{\alpha}}} \nabla_{\mathbf{r}_{1}} \beta u_{a_{\mu_{1} \ldots a_{\mu_{p}} a_{\nu_{1}} \ldots a_{\nu_{\alpha}}}}=0,
\end{gathered}
$$


where the higher distribution functions $f_{a_{1} \ldots a_{s} a_{\nu_{1}} \ldots a_{\nu_{\alpha}}}$ for $s+\alpha$ particles have been introduced under the $3 \alpha$-fold integral; to be precise, similar to equation (2) for $\alpha<N-s$ and $f_{a_{1} \ldots a_{N}}=\left(V^{N} / Q_{N}\right) \exp \left(-\beta w_{a_{1} \ldots a_{N}}\right)$ for $\alpha=N-s$, i. e., equation (9) is a coupled set of integro-differential equations that exactly determines $f_{a_{1} \ldots a_{s}}$ for $2 \leqslant s \leqslant N-1$. While in the case of mere two-body interaction the two-particle distribution couples to that for three particles, and so on, the general many-body interaction, equation (1), produces a coupling of $f_{a_{1} \ldots a_{s}}$ to the whole sequence of all higher distribution functions.

Usually one is only interested in the distributions of few particles up to, let's say, $s=4$ and, therefore, wants to solve equation (9) for $s \ll N$. On this condition we can extend the range of the $\alpha$ summations over the $\nu$ 's from $s+1 \ldots N$ to $1 \ldots N$, and then apply the following relation for any function $\mathcal{G}_{a_{1} a_{2} \ldots}$, that solely depends on the species, to replace sums over particle numbers with sums over particle species,

$$
\sum_{\lambda_{1} \neq \ldots \neq \lambda_{\alpha}=1}^{N} \mathcal{G}_{a_{\lambda_{1}} \ldots a_{\lambda_{\alpha}}}=\sum_{a_{1}, \ldots, a_{\alpha}} N_{a_{1}} \ldots N_{a_{\alpha}} \mathcal{G}_{a_{1} \ldots a_{\alpha}}+O\left(N^{\alpha-1}\right)
$$

e.g.,

$$
\begin{aligned}
\sum_{i=1}^{N} \mathcal{G}_{a_{i}} & =\sum_{a} N_{a} \mathcal{G}_{a} \\
\sum_{i \neq j=1}^{N} \mathcal{G}_{a_{i} a_{j}} & =\sum_{a, b} N_{a} N_{b} \mathcal{G}_{a b}-\sum_{a} N_{a} \mathcal{G}_{a a} \\
\sum_{i \neq j \neq k=1}^{N} \mathcal{G}_{a_{i} a_{j} a_{k}} & =\sum_{a, b, c} N_{a} N_{b} N_{c} \mathcal{G}_{a b c}-3 \sum_{a, b} N_{a} N_{b} \mathcal{G}_{a a b}+2 \sum_{a} N_{a} \mathcal{G}_{a a a}
\end{aligned}
$$

The contributions from equation (10) of the orders $N^{\alpha-1}, N^{\alpha-2}, \ldots, N$ will vanish when they are divided by $V^{\alpha}$ and the thermodynamic limit is performed for $N_{a}, V \rightarrow$ $\infty$, whereas the partial densities $n_{a}=N_{a} / V$ remain finite. In this way one arrives at the famous BBGKY hierarchy of equations for $f_{a_{1} \ldots a_{s}}$ in the generalized version

$$
\begin{aligned}
& \nabla_{\mathbf{r}_{1}} f_{a_{1} \ldots a_{s}}+f_{a_{1} \ldots a_{s}} \nabla_{\mathbf{r}_{1}} \beta w_{a_{1} \ldots a_{s}}+\sum_{\alpha=1}^{N-s} \frac{1}{\alpha !} \sum_{a_{s+1}} \ldots \sum_{a_{s+\alpha}} n_{a_{s+1}} \ldots n_{a_{s+\alpha}} \\
& \quad \times \int \mathrm{d} \mathbf{r}_{s+1} \ldots \mathrm{d} \mathbf{r}_{s+\alpha} f_{a_{1} \ldots a_{s+\alpha}} \nabla_{\mathbf{r}_{1}} \beta w_{a_{1} \ldots a_{s}, a_{s+1} \ldots a_{s+\alpha}}=0
\end{aligned}
$$

which takes into account many-body interactions. The second term in equation (14) comes from the direct interaction among the $s$ particles via $w_{a_{1} \ldots a_{s}}$, the third term describes the collective influence of the $N-s$ particles on the former, where the potential

$$
w_{a_{1} \ldots a_{s}, a_{s+1} \ldots a_{s+\alpha}}=\sum_{p=1}^{s} \sum_{\mu_{1}<\cdots<\mu_{p}=1}^{s} u_{a_{\mu_{1} \ldots a_{\mu_{p}} a_{s+1} \ldots a_{s+\alpha}}}
$$


differs from equation (8) in that the $\alpha$ particles are now those of species $a_{s+1}, \ldots, a_{s+\alpha}$ at positions $\mathbf{r}_{s+1}, \ldots, \mathbf{r}_{s+\alpha}$ and no longer a varying choice from the $N-s$ particles,

$$
\begin{aligned}
w_{a_{1} \ldots a_{s}, a_{s+1}} & =\sum_{i=1}^{s} u_{a_{i} a_{s+1}}+\sum_{i<j=1}^{s} u_{a_{i} a_{j} a_{s+1}}+\cdots+u_{a_{1} \ldots a_{s} a_{s+1}}, \\
w_{a_{1} \ldots a_{s}, a_{s+1} a_{s+2}} & =\sum_{i=1}^{s} u_{a_{i} a_{s+1} a_{s+2}}+\cdots+u_{a_{1} \ldots a_{s} a_{s+1} a_{s+2}}, \\
& \vdots \\
w_{a_{1} \ldots a_{s}, a_{s+1} \ldots a_{N}} & =\sum_{i=1}^{s} u_{a_{i} a_{s+1} \ldots a_{N}}+\sum_{i<j=1}^{s} u_{a_{i} a_{j} a_{s+1} \ldots a_{N}}+\cdots+u_{a_{1} \ldots a_{N}} .
\end{aligned}
$$

Though equation (14) has been derived for small $s$, the distribution $f_{a_{1} \ldots a_{s}}$ nevertheless couples to $f_{a_{1} \ldots a_{s+1}}, \ldots, f_{a_{1} \ldots a_{N}}$ as in equation (9), but seeing that $f_{a_{1} \ldots a_{s+\alpha}}$ is associated with the density power $n^{\alpha}$, one might hope to decouple the hierarchy by expanding the distribution functions at low densities. Such a procedure will only work without difficulty if all the interactions are strictly short-ranged, i. e., the potentials drop off more rapidly than $1 / r_{i j}^{3}$ for $r_{i j} \equiv\left|\mathbf{r}_{i}-\mathbf{r}_{j}\right| \rightarrow \infty$; otherwise some of the resulting integrals will diverge in the thermodynamic limit. In the system under consideration, however, each particle of species $a$ bears a charge $q_{a}$, and so the most characteristic type of interaction is the long-range, two-body Coulomb interaction via the potential $v_{a b}\left(r_{i j}\right)=q_{a} q_{b} / r_{i j}$, which is part of $u_{a b}$ and, hence, also of $w_{a_{1} \ldots a_{s}}$ and $w_{a_{1} \ldots a_{s}, a_{s+1}}$. Because the higher components $u_{a b c}, u_{a b c d}, \ldots$ are supposed to be short-ranged, $v_{a b}$ is the only long-range potential at all, and $u_{a b}=v_{a b}+v_{a b}^{\prime}$ can be written as the sum of $v_{a b}$ and a short-range part $v_{a b}^{\prime}$. In the same manner, we split up $w_{a_{1} \ldots a_{s}}$ and $w_{a_{1} \ldots a_{s}, a_{s+1}}$, thereby defining the short-range potentials $w_{a_{1} \ldots a_{s}}^{\prime}$ and $w_{a_{1} \ldots a_{s}, a_{s+1}}^{\prime}$,

$$
\begin{aligned}
w_{a_{1} \ldots a_{s}} & =\sum_{i<j=1}^{s} v_{a_{i} a_{j}}+w_{a_{1} \ldots a_{s}}^{\prime}, \\
w_{a_{1} \ldots a_{s}}^{\prime} & =\sum_{i<j=1}^{s} v_{a_{i} a_{j}}^{\prime}+\sum_{i<j<k=1}^{s} u_{a_{i} a_{j} a_{k}}+\cdots+u_{a_{1} \ldots a_{s}}, \\
w_{a_{1} \ldots a_{s}, a_{s+1}} & =\sum_{i=1}^{s} v_{a_{i} a_{s+1}}+w_{a_{1} \ldots a_{s}, a_{s+1}}^{\prime}, \\
w_{a_{1} \ldots a_{s}, a_{s+1}}^{\prime} & =\sum_{i=1}^{s} v_{a_{i} a_{s+1}}^{\prime}+\sum_{i<j=1}^{s} u_{a_{i} a_{j} a_{s+1}}+\cdots+u_{a_{1} \ldots a_{s} a_{s+1}} .
\end{aligned}
$$

At this point of our derivation, screening of the Coulomb potential is introduced, for which we will utilize the following integral equation [1] in two opposite directions,

$$
\beta v_{a b}-\beta \tilde{v}_{a b}-\sum_{c} n_{c} \int \mathrm{d} \mathbf{r}_{3} \beta v_{a c} \beta \tilde{v}_{b c}=0,
$$


that relates the bare potential $v_{a b}$ and the screened potential $\tilde{v}_{a b}$ with each other. The traditional "forward" use [9] consists in converting equation (23) into a partial differential equation, the linearized Poisson-Boltzmann equation, and solving it by the Fourier transform method to get the well-known expression $\tilde{v}_{a b}\left(r_{i j}\right)=$ $q_{a} q_{b} \exp \left(-\kappa r_{i j}\right) / r_{i j}$, where the Debye-Hückel parameter $\kappa=\sqrt{4 \pi \beta \sum_{a} n_{a} q_{a}^{2}}$ plays the role of the inverse classical screening length, as usual. Vice versa, one may regard $\tilde{v}_{a b}$ as given quantity and go "backwards" to substitute for the Coulomb potential occurring twice in the BBGKY hierarchy, equation (14), the series expansion $\beta v_{a b}=\lim _{N \rightarrow \infty} \sum_{m=0}^{N-2} G_{a b}^{(m)}[3,10]$, the terms of which are found from an iteration of the screening equation, equation (23),

$$
G_{a b}^{(0)}=-g_{a b}, \quad G_{a b}^{(m)}=-\sum_{c} n_{c} \int \mathrm{d} \mathbf{r}_{3} G_{a c}^{(m-1)} g_{b c}, \quad m>0
$$

so that

$$
\beta v_{a b}=-g_{a b}+\sum_{c} n_{c} \int \mathrm{d} \mathbf{r}_{3} g_{a c} g_{b c}-\sum_{c, d} n_{c} n_{d} \int \mathrm{d} \mathbf{r}_{3} \mathrm{~d} \mathbf{r}_{4} g_{a c} g_{b d} g_{c d}+\cdots,
$$

with $g_{a b}=-\beta \tilde{v}_{a b}$ being the correlation function in the Debye-Hückel approximation.

In the limit of infinite dilution, $n_{a} \rightarrow 0$ for all species, the hierarchy reduces to the first two terms of equation (14) and then has the simple solution $f_{a_{1} \ldots a_{s}} \propto$ $\exp \left(-\beta w_{a_{1} \ldots a_{s}}\right)$. This suggests constructing an ansatz for $f_{a_{1} \ldots a_{s}}$ at finite density in the form [9]

$$
f_{a_{1} \ldots a_{s}}=C_{a_{1} \ldots a_{s}} \exp \left[-\beta\left(\sum_{i<j=1}^{s} \tilde{v}_{a_{i} a_{j}}+w_{a_{1} \ldots a_{s}}^{\prime}\right)\right],
$$

where screening directly enters through $\tilde{v}_{a b}$ that replaces $v_{a b}=\lim _{\kappa \rightarrow 0} \tilde{v}_{a b}$ in $w_{a_{1} \ldots a_{s}}$, equation (19), and other collective effects are handled by allowing the coefficient $C_{a_{1} \ldots a_{s}}$ to depend on the particles' densities under the constraint $\lim _{n_{a} \rightarrow 0} C_{a_{1} \ldots a_{s}}=1$. Inserting equation (26) into the full BBGKY hierarchy, with the term for $\alpha=1$ taken separately, and dividing by the exponential with the help of $w_{a_{1} \ldots a_{s+1}}^{\prime}-w_{a_{1} \ldots a_{s}}^{\prime}=$ $w_{a_{1} \ldots a_{s}, a_{s+1}}^{\prime}$ and

$$
w_{a_{1} \ldots a_{s+\alpha}}^{\prime}-w_{a_{1} \ldots a_{s}}^{\prime}=w_{a_{s+1} \ldots a_{s+\alpha}}^{\prime}+\sum_{\lambda=s+1}^{s+\alpha} w_{a_{1} \ldots a_{s}, a_{\lambda}}^{\prime}+\sum_{p=2}^{\alpha} \sum_{\lambda_{1}<\cdots<\lambda_{p}=s+1}^{s+\alpha} w_{a_{1} \ldots a_{s}, a_{\lambda_{1}} \ldots a_{\lambda_{p}}}
$$

for $\alpha>1$, leads to coupled equations for $C_{a_{1} \ldots a_{s}}$ which are free of the bare potential,

$$
\begin{aligned}
& \nabla_{\mathbf{r}_{1}} C_{a_{1} \ldots a_{s}}+C_{a_{1} \ldots a_{s}} \nabla_{\mathbf{r}_{1}} \sum_{m=1}^{N-s} \sum_{i<j=1}^{s} G_{a_{i} a_{j}}^{(m)}+\sum_{a_{s+1}} n_{a_{s+1}} \int \mathrm{d} \mathbf{r}_{s+1} C_{a_{1} \ldots a_{s+1}} \\
& \quad \times \exp \left(-\beta \tilde{w}_{a_{1} \ldots a_{s}, a_{s+1}}\right) \nabla_{\mathbf{r}_{1}}\left(\sum_{m=1}^{N-s-1} \sum_{i=1}^{s} G_{a_{i} a_{s+1}}^{(m)}+\beta \tilde{w}_{a_{1} \ldots a_{s}, a_{s+1}}\right)
\end{aligned}
$$




$$
\begin{aligned}
& +\sum_{\alpha=2}^{N-s} \frac{1}{\alpha !} \sum_{a_{s+1}} \ldots \sum_{a_{s+\alpha}} n_{a_{s+1}} \ldots n_{a_{s+\alpha}} \int \mathrm{d} \mathbf{r}_{s+1} \ldots \mathrm{d} \mathbf{r}_{s+\alpha} C_{a_{1} \ldots a_{s+\alpha}} \\
& \times \exp \left[-\beta\left(\sum_{\lambda=s+1}^{s+\alpha} \tilde{w}_{a_{1} \ldots a_{s}, a_{\lambda}}+\sum_{p=2}^{\alpha} \sum_{\lambda_{1}<\cdots<\lambda_{p}=s+1}^{s+\alpha} w_{a_{1} \ldots a_{s}, a_{\lambda_{1}} \ldots a_{\lambda_{p}}}\right.\right. \\
& \left.\left.+\tilde{w}_{a_{s+1} \ldots a_{s+\alpha}}\right)\right] \nabla_{\mathbf{r}_{1}} \beta w_{a_{1} \ldots a_{s}, a_{s+1} \ldots a_{s+\alpha}}=0 .
\end{aligned}
$$

The screened composite potentials in equation (27), $\tilde{w}_{a_{s+1} \ldots a_{s+\alpha}}$ and $\tilde{w}_{a_{1} \ldots a_{s}, a_{s+1}}$, originate from the unscreened ones, $w_{a_{s+1} \ldots a_{s+\alpha}}$ and $w_{a_{1} \ldots a_{s}, a_{s+1}}$, with $\tilde{v}_{a b}$ instead of $v_{a b}$,

$$
\begin{aligned}
\tilde{w}_{a_{s+1} \ldots a_{s+\alpha}} & =\sum_{i<j=s+1}^{s+\alpha} \tilde{v}_{a_{i} a_{j}}+w_{a_{s+1} \ldots a_{s+\alpha}}^{\prime} \\
& =\sum_{i<j=s+1}^{s+\alpha} \tilde{u}_{a_{i} a_{j}}+\sum_{i<j<k=s+1}^{s+\alpha} u_{a_{i} a_{j} a_{k}}+\cdots+u_{a_{s+1} \ldots a_{s+\alpha}}
\end{aligned}
$$

and

$$
\begin{aligned}
\tilde{w}_{a_{1} \ldots a_{s}, a_{s+1}} & =\sum_{i=1}^{s} \tilde{v}_{a_{i} a_{s+1}}+w_{a_{1} \ldots a_{s}, a_{s+1}}^{\prime} \\
& =\sum_{i=1}^{s} \tilde{u}_{a_{i} a_{s+1}}+\sum_{i<j=1}^{s} u_{a_{i} a_{j} a_{s+1}}+\cdots+u_{a_{1} \ldots a_{s} a_{s+1}}
\end{aligned}
$$

where the two-body potential $\tilde{u}_{a b}=\tilde{v}_{a b}+v_{a b}^{\prime}$ is the screened analogue to $u_{a b}$.

\section{The virial expansion of the distribution functions}

As we learnt in the preceding section, equation (27) holds for a many-particle system with two-body Coulomb interaction and additional short-range interactions between two and more particles. Since screening has removed all long-range components, the hierarchy now only contains screened and short-range potentials, $\tilde{v}_{a b}$, $v_{a b}^{\prime}, u_{a b c}, \ldots$, and thus can be decoupled by doing the virial expansion $C_{a_{1} \ldots a_{s}}=$ $\sum_{m=0}^{N-s} C_{a_{1} \ldots a_{s}}^{(m)}$ that is very similar to the one above for $\beta v_{a b}$. A careful analysis of its structure clearly reveals that the coefficient $C_{a_{1} \ldots a_{s}}^{(m)}$, for $m>0$, includes the factor $n_{a_{s+1}} \ldots n_{a_{s+m}}$, i. e., disregarding at the moment the functional dependence upon $\kappa$, it is formally of the density order $n^{m}$, and the same correspondingly applies to $G_{a b}^{(m)}$. This provides the following recipe how one obtains an equation for $C_{a_{1} \ldots a_{s}}^{(k)}$ : introduce the auxiliary parameter $\gamma$ and assign it to each density in equation (27) occurring as a factor (i. e., not to $\kappa$ ), multiply the terms and collect up those which belong to $\gamma^{k}$. The result reads

$$
\nabla_{\mathbf{r}_{1}} C_{a_{1} \ldots a_{s}}^{(k)}+\sum_{m=1}^{k}\left(\nabla_{\mathbf{r}_{1}} G_{a_{1} \ldots a_{s}}^{(m)}\right) C_{a_{1} \ldots a_{s}}^{(k-m)}
$$




$$
\begin{aligned}
& -\sum_{a_{s+1}} n_{a_{s+1}} \int \mathrm{d} \mathbf{r}_{s+1}\left[\left(\nabla_{\mathbf{r}_{1}} H_{a_{1} \ldots a_{s}, a_{s+1}}\right) C_{a_{1} \ldots a_{s+1}}^{(k-1)}\right. \\
& \left.-\sum_{m=1}^{k-1}\left(\nabla_{\mathbf{r}_{1}} G_{a_{1} \ldots a_{s}, a_{s+1}}^{(m)}\right)\left(H_{a_{1} \ldots a_{s}, a_{s+1}}+1\right) C_{a_{1} \ldots a_{s+1}}^{(k-1-m)}\right] \\
& -\sum_{\alpha=2}^{k} \frac{1}{\alpha !} \sum_{a_{s+1}} \ldots \sum_{a_{s+\alpha}} n_{a_{s+1}} \ldots n_{a_{s+\alpha}} \int \mathrm{d} \mathbf{r}_{s+1} \ldots \mathrm{d} \mathbf{r}_{s+\alpha}\left(\nabla_{\mathbf{r}_{1}} H_{a_{1} \ldots a_{s}, a_{s+1} \ldots a_{s+\alpha}}\right) \\
& \times \prod_{p=1}^{\alpha-1} \prod_{\lambda_{1}<\cdots<\lambda_{p}=s+1}^{s+\alpha}\left(H_{a_{1} \ldots a_{s}, a_{\lambda_{1}} \ldots a_{\lambda_{p}}}+1\right)\left(H_{a_{s+1} \ldots a_{s+\alpha}}+1\right) C_{a_{1} \ldots a_{s+\alpha}}^{(k-\alpha)}=0
\end{aligned}
$$

and has been cast in this highly compact form by using sums over $G_{a b}^{(m)}$, equation $(24)$,

$$
G_{a_{1} \ldots a_{s}}^{(m)}=\sum_{i<j=1}^{s} G_{a_{i} a_{j}}^{(m)} \quad \text { and } \quad G_{a_{1} \ldots a_{s}, a_{s+1}}^{(m)}=\sum_{i=1}^{s} G_{a_{i} a_{s+1}}^{(m)},
$$

and Mayer cluster functions for the composite potentials, equations (29), (31), (15), $\alpha>1$,

$$
\begin{aligned}
H_{a_{s+1} \ldots a_{s+\alpha}} & =\exp \left(-\beta \tilde{w}_{a_{s+1} \ldots a_{s+\alpha}}\right)-1 \\
H_{a_{1} \ldots a_{s}, a_{s+1}} & =\exp \left(-\beta \tilde{w}_{a_{1} \ldots a_{s}, a_{s+1}}\right)-1 \\
H_{a_{1} \ldots a_{s}, a_{s+1} \ldots a_{s+\alpha}} & =\exp \left(-\beta w_{a_{1} \ldots a_{s}, a_{s+1} \ldots a_{s+\alpha}}\right)-1 .
\end{aligned}
$$

Unlike the truncated equation (3.6) in [3], equation (32) displays in its last two lines the general term needed for the calculation of $C_{a_{1} \ldots a_{s}}^{(k)}, k>2$, and therefore yields, at least in principle, the complete series of virial coefficients for $s$ particles at the order $k=0, \ldots, N-s$. As a consequence of the screened virial, or cluster, expansion of $C_{a_{1} \ldots a_{s}}$, the sum over $\alpha$ is now cut off at $k$, and so $C_{a_{1} \ldots a_{s}}^{(k)}$ couples to the coefficients for at most $s+k$ particles whose order is lowered to $k-1, k-2, \ldots, 0$. Then, it is obvious to solve equation (32) step by step from $k=0$ to the desired order, where the solution must satisfy the boundary condition for vanishing particle correlations at infinity, $\lim _{r_{\mu \nu} \rightarrow \infty} C_{a_{1} \ldots a_{s}}^{(k)}=\delta_{k, 0}(\mu<\nu=1, \ldots, s)$. At zeroth order, $k=0$, equation (32) collapses to $\nabla_{\mathbf{r}_{1}} C_{a_{1} \ldots a_{s}}^{(0)}=0$, and in view of the boundary condition we have at once $C_{a_{1} \ldots a_{s}}^{(0)}=1$.

Although equation (32) would also permit to be treated at higher orders without too much effort, we will restrict ourselves to the first two non-trivial coefficients $C_{a_{1} \ldots a_{s}}^{(1)}$ and $C_{a_{1} \ldots a_{s}}^{(2)}$. Let $k=1$, and the first-order coefficient $C_{a_{1} \ldots a_{s}}^{(1)}$ obeys the equation

$$
\nabla_{\mathbf{r}_{1}} C_{a_{1} \ldots a_{s}}^{(1)}+\nabla_{\mathbf{r}_{1}}\left(G_{a_{1} \ldots a_{s}}^{(1)}-\sum_{a_{s+1}} n_{a_{s+1}} \int \mathrm{d} \mathbf{r}_{s+1} H_{a_{1} \ldots a_{s}, a_{s+1}}\right)=0
$$

from which the result of integration is evident while the integration constant can be derived as follows. We consider the integral over the cluster function $H_{a_{1} \ldots a_{s}, a_{s+1}}$, 
equation (35), with respect to $\mathbf{r}_{s+1}$, depending on the $\left(\begin{array}{l}s \\ 2\end{array}\right)=\frac{1}{2} s(s-1)$ relative coordinates $\left\{r_{i j}\right\}=r_{12}, \ldots, r_{s-1 s}$ that are simultaneously sent to infinity. In this limit, all the component potentials $u_{a_{i} a_{j} \ldots a_{s+1}}$ in $\tilde{w}_{a_{1} \ldots a_{s}, a_{s+1}}$ with at least two variables $\mathbf{r}_{i}, \mathbf{r}_{j}, \ldots$ do not contribute, i. e., only the first sum in equation (31) remains and $\int \mathrm{d} \mathbf{r}_{s+1} H_{a_{1} \ldots a_{s}, a_{s+1}}$ approaches $\int \mathrm{d} \mathbf{r}_{s+1} \hat{H}_{a_{1} \ldots a_{s}, a_{s+1}}$, where we meet the (asymptotic) cluster function

$$
\hat{H}_{a_{1} \ldots a_{s}, a_{s+1}}=\exp \left(-\beta \sum_{i=1}^{s} \tilde{u}_{a_{i} a_{s+1}}\right)-1 .
$$

According to Guernsey [11], one can identically rewrite $\hat{H}_{a_{1} \ldots a_{s}, a_{s+1}}$ in terms of products of Mayer functions $h_{a b}=\exp \left(-\beta \tilde{u}_{a b}\right)-1$ for the screened pair potential $\tilde{u}_{a b}$,

$$
\hat{H}_{a_{1} \ldots a_{s}, a_{s+1}}=\sum_{i=1}^{s} h_{a_{i} a_{s+1}}+\sum_{i<j=1}^{s} h_{a_{i} a_{s+1}} h_{a_{j} a_{s+1}}+\cdots+h_{a_{1} a_{s+1}} h_{a_{2} a_{s+1}} \ldots h_{a_{s} a_{s+1}} .
$$

Due to the localization of $h_{a_{i} a_{s+1}}$ around $\mathbf{r}_{i}$, the overlap between the different $h$ 's in equation (39) vanishes at infinite particle separation, $r_{i j} \rightarrow \infty$, and again only the first sum is left over under the integral. Putting these steps together, we conclude that

$$
\lim _{r_{\mu \nu} \rightarrow \infty} \int \mathrm{d} \mathbf{r}_{s+1} H_{a_{1} \ldots a_{s}, a_{s+1}}=\int \mathrm{d} \mathbf{r}_{s+1} \sum_{i=1}^{s} h_{a_{i} a_{s+1}}=\text { const },
$$

which is indeed a constant because of the spherical symmetry of the last integrand. The other term in equation (37), $G_{a_{1} \ldots a_{s}}^{(1)}$, gives no contribution to the integration constant for the same reason as before, and, with equation (40), $C_{a_{1} \ldots a_{s}}^{(1)}$ eventually becomes

$$
C_{a_{1} \ldots a_{s}}^{(1)}=\sum_{a_{s+1}} n_{a_{s+1}} \int \mathrm{d} \mathbf{r}_{s+1}\left(H_{a_{1} \ldots a_{s}, a_{s+1}}-\sum_{i=1}^{s} h_{a_{i} a_{s+1}}\right)-G_{a_{1} \ldots a_{s}}^{(1)} .
$$

The determination of $C_{a_{1} \ldots a_{s}}^{(2)}$, the coefficient at second order, is more difficult as expected, and requires some additional steps. First, we specify equation (32) for $k=2$,

$$
\begin{aligned}
& \nabla_{\mathbf{r}_{1}} C_{a_{1} \ldots a_{s}}^{(2)}+\left(\nabla_{\mathbf{r}_{1}} G_{a_{1} \ldots a_{s}}^{(1)}\right) C_{a_{1} \ldots a_{s}}^{(1)}+\nabla_{\mathbf{r}_{1}} G_{a_{1} \ldots a_{s}}^{(2)}-\sum{ }_{a_{s+1}} n_{a_{s+1}} \int \mathrm{d} \mathbf{r}_{s+1} \\
& \times\left[\left(\nabla_{\mathbf{r}_{1}} H_{a_{1} \ldots a_{s}, a_{s+1}}\right) C_{a_{1} \ldots a_{s+1}}^{(1)}-\left(\nabla_{\mathbf{r}_{1}} G_{a_{1} \ldots a_{s}, a_{s+1}}^{(1)}\right) H_{a_{1} \ldots a_{s}, a_{s+1}}\right] \\
& -\frac{1}{2} \sum_{a_{s+1}} \sum_{a_{s+2}} n_{a_{s+1}} n_{a_{s+2}} \int \mathrm{d} \mathbf{r}_{s+1} \mathrm{~d} \mathbf{r}_{s+2}\left(\nabla_{\mathbf{r}_{1}} H_{a_{1} \ldots a_{s}, a_{s+1} a_{s+2}}\right) \\
& \times\left(H_{a_{1} \ldots a_{s}, a_{s+1}}+1\right)\left(H_{a_{1} \ldots a_{s}, a_{s+2}}+1\right)\left(h_{a_{s+1} a_{s+2}}+1\right)=0,
\end{aligned}
$$

where $\int \mathrm{d} \mathbf{r}_{s+1} G_{a_{1} \ldots a_{s}, a_{s+1}}^{(1)}=$ const and $H_{a_{s+1} a_{s+2}}=h_{a_{s+1} a_{s+2}}$ have been used, cf. equations (33), (40), and (34). With equation (41) inserted for $C_{a_{1} \ldots a_{s}}^{(1)}$, and with the 
two relations

$$
\begin{aligned}
& H_{a_{1} \ldots a_{s+1}, a_{s+2}}-h_{a_{s+1} a_{s+2}}= \\
& \quad=H_{a_{1} \ldots a_{s}, a_{s+1} a_{s+2}}\left(H_{a_{1} \ldots a_{s}, a_{s+2}}+1\right)\left(h_{a_{s+1} a_{s+2}}+1\right)+H_{a_{1} \ldots a_{s}, a_{s+2}}\left(h_{a_{s+1} a_{s+2}}+1\right)
\end{aligned}
$$

and $G_{a_{1} \ldots a_{s}}^{(1)}+G_{a_{1} \ldots a_{s}, a_{s+1}}^{(1)}=G_{a_{1} \ldots a_{s+1}}^{(1)}$, we can bring equation (42) to the integrable form

$$
\begin{aligned}
& \nabla_{\mathbf{r}_{1}} C_{a_{1} \ldots a_{s}}^{(2)}+\nabla_{\mathbf{r}_{1}}\left\{G_{a_{1} \ldots a_{s}}^{(2)}-\frac{1}{2}\left(G_{a_{1} \ldots a_{s}}^{(1)}\right)^{2}\right. \\
& +\sum_{a_{s+1}} n_{a_{s+1}} \int \mathrm{d} \mathbf{r}_{s+1}\left(G_{a_{1} \ldots a_{s+1}}^{(1)} H_{a_{1} \ldots a_{s}, a_{s+1}}-G_{a_{1} \ldots a_{s}}^{(1)} \sum_{i=1}^{s} h_{a_{i} a_{s+1}}\right) \\
& -\frac{1}{2} \sum_{a_{s+1}} \sum_{a_{s+2}} n_{a_{s+1}} n_{a_{s+2}} \int \mathrm{d} \mathbf{r}_{s+1} \mathrm{~d} \mathbf{r}_{s+2} \\
& \times\left[H_{a_{1} \ldots a_{s}, a_{s+1} a_{s+2}}\left(H_{a_{1} \ldots a_{s}, a_{s+1}}+1\right)\left(H_{a_{1} \ldots a_{s}, a_{s+2}}+1\right)\left(h_{a_{s+1} a_{s+2}}+1\right)\right. \\
& \quad+H_{a_{1} \ldots a_{s}, a_{s+1}} H_{a_{1} \ldots a_{s}, a_{s+2}}\left(h_{a_{s+1} a_{s+2}}+1\right) \\
& \left.\left.\quad-H_{a_{1} \ldots a_{s}, a_{s+1}} \sum_{i=1}^{s} h_{a_{i} a_{s+2}}-H_{a_{1} \ldots a_{s}, a_{s+2}} \sum_{i=1}^{s} h_{a_{i} a_{s+1}}\right]\right\}=0
\end{aligned}
$$

the correctness of which is most easily verified backwards: taking the derivative in equation (43), employing equation (40) and the symmetry of the terms in the square brackets against the interchange $s+1 \leftrightarrow s+2$, we get a complete agreement with equation (42). The rest of the calculation is then almost as straightforward as for $C_{a_{1} \ldots a_{s}}^{(1)}$, and the same arguments as above lead to some new contributions to the integration constant,

$$
\begin{aligned}
& \lim _{r_{\mu \nu} \rightarrow \infty} \int \mathrm{d} \mathbf{r}_{s+1} G_{a_{1} \ldots a_{s}, a_{s+1}}^{(1)} H_{a_{1} \ldots a_{s}, a_{s+1}}=\int \mathrm{d} \mathbf{r}_{s+1} \sum_{i=1}^{s} G_{a_{i} a_{s+1}}^{(1)} h_{a_{i} a_{s+1}}, \\
& \lim _{r_{\mu \nu} \rightarrow \infty} \int \mathrm{d} \mathbf{r}_{s+1} \mathrm{~d} \mathbf{r}_{s+2} H_{a_{1} \ldots a_{s}, a_{s+1} a_{s+2}} \\
& \times\left(H_{a_{1} \ldots a_{s}, a_{s+1}}+1\right)\left(H_{a_{1} \ldots a_{s}, a_{s+2}}+1\right)\left(h_{a_{s+1} a_{s+2}}+1\right) \\
& =\int \mathrm{d} \mathbf{r}_{s+1} \mathrm{~d} \mathbf{r}_{s+2}\left(h_{a_{s+1} a_{s+2}}+1\right) \sum_{i=1}^{s} h_{a_{i} a_{s+1} a_{s+2}}\left(h_{a_{i} a_{s+1}}+1\right)\left(h_{a_{i} a_{s+2}}+1\right), \\
& \lim _{r_{\mu \nu} \rightarrow \infty} \int \mathrm{d} \mathbf{r}_{s+1} \mathrm{~d} \mathbf{r}_{s+2} H_{a_{1} \ldots a_{s}, a_{s+1}} H_{a_{1} \ldots a_{s}, a_{s+2}}\left(h_{a_{s+1} a_{s+2}}+1\right) \\
& =\int \mathrm{d} \mathbf{r}_{s+1} \mathrm{~d} \mathbf{r}_{s+2}\left(h_{a_{s+1} a_{s+2}} \sum_{i=1}^{s} h_{a_{i} a_{s+1}} h_{a_{i} a_{s+2}}+\sum_{i=1}^{s} h_{a_{i} a_{s+1}} \sum_{j=1}^{s} h_{a_{j} a_{s+2}}\right),
\end{aligned}
$$

besides those, from the last two terms in equation (43), already familiar from equation (40), with the three-particle Mayer function $h_{a b c}=\exp \left(-\beta u_{a b c}\right)-1$. The final 
result is

$$
\begin{aligned}
& C_{a_{1} \ldots a_{s}}^{(2)}=\frac{1}{2}\left(C_{a_{1} \ldots a_{s}}^{(1)}\right)^{2}-\sum_{a_{s+1}} n_{a_{s+1}} \int \mathrm{d} \mathbf{r}_{s+1}\left(G_{a_{1} \ldots a_{s}, a_{s+1}}^{(1)} H_{a_{1} \ldots a_{s}, a_{s+1}}-\sum_{i=1}^{s} G_{a_{i} a_{s+1}}^{(1)} h_{a_{i} a_{s+1}}\right) \\
& +\frac{1}{2} \sum_{a_{s+1}} \sum_{a_{s+2}} n_{a_{s+1}} n_{a_{s+2}} \int \mathrm{d} \mathbf{r}_{s+1} \mathrm{~d} \mathbf{r}_{s+2} \\
& \times\left\{\left[H_{a_{1} \ldots a_{s}, a_{s+1} a_{s+2}}\left(H_{a_{1} \ldots a_{s}, a_{s+1}}+1\right)\left(H_{a_{1} \ldots a_{s}, a_{s+2}}+1\right)\right.\right. \\
& \left.\quad-\sum_{i=1}^{s} h_{a_{i} a_{s+1} a_{s+2}}\left(h_{a_{i} a_{s+1}}+1\right)\left(h_{a_{i} a_{s+2}}+1\right)\right]\left(h_{a_{s+1} a_{s+2}}+1\right) \\
& \left.+h_{a_{s+1} a_{s+2}}\left(H_{a_{1} \ldots a_{s}, a_{s+1}} H_{a_{1} \ldots a_{s}, a_{s+2}}-\sum_{i=1}^{s} h_{a_{i} a_{s+1}} h_{a_{i} a_{s+2}}\right)\right\}-G_{a_{1} \ldots a_{s}}^{(2)},
\end{aligned}
$$

where $C_{a_{1} \ldots a_{s}}^{(1)}$, equation (41), has been reintroduced to make this expression shorter.

\section{Summary and comparisons}

To summarize our paper, we derived the screened BBGKY hierarchy of equations, equation (27), for the reduced distribution of $s$ charged, classical particles with longrange and many-body short-range interactions, and solved it for the first coefficients in the cluster expansion of $f_{a_{1} \ldots a_{s}}$ through second order, so that the calculation ends in

$$
f_{a_{1} \ldots a_{s}}=\left(1+C_{a_{1} \ldots a_{s}}^{(1)}+C_{a_{1} \ldots a_{s}}^{(2)}\right) \exp \left(-\beta \tilde{w}_{a_{1} \ldots a_{s}}\right)
$$

The full expression (41) for $C_{a_{1} \ldots a_{s}}^{(1)}$ was obtained by Schmitz, equation (3.10) in [3], more than thirty years ago, but in contrast he failed in getting an equivalent result for $C_{a_{1} \ldots a_{s}}^{(2)}$, which was given by the present author only recently, see equation (6) and the discussion in [7]. We, therefore, regard the derivation of $C_{a_{1} \ldots a_{s}}^{(2)}$, equation (47), in the last section as the first complete approach to the distribution function at second order for the described physical system based on the BBGKY hierarchy.

Examples of the coefficients for $s=2$ and 3, namely $C_{a b}^{(1)}, C_{a b}^{(2)}$, and $C_{a b c}^{(1)}$, can be found in [12] and, in part, compared with Friedman's corresponding contributions in graphical representation [9] as far as they are shown in his figure 14.1. In the special case of pairwise interactions, $u_{a_{1} \ldots a_{\lambda}} \equiv 0$ if $\lambda>2$, our expressions for $C_{a_{1} \ldots a_{s}}^{(1)}$ and $C_{a_{1} \ldots a_{s}}^{(2)}$ become identical to Schmitz' earlier results [13] as it should be; for the sake of convenience they are repeated here in our notation, especially with equation (38),

$$
\begin{aligned}
& \hat{C}_{a_{1} \ldots a_{s}}^{(1)}=\sum_{a_{s+1}} n_{a_{s+1}} \int \mathrm{d} \mathbf{r}_{s+1}\left(\hat{H}_{a_{1} \ldots a_{s}, a_{s+1}}-\sum_{i=1}^{s} h_{a_{i} a_{s+1}}\right)-G_{a_{1} \ldots a_{s}}^{(1)}, \\
& \hat{C}_{a_{1} \ldots a_{s}}^{(2)}=\frac{1}{2}\left(\hat{C}_{a_{1} \ldots a_{s}}^{(1)}\right)^{2}-G_{a_{1} \ldots a_{s}}^{(2)}
\end{aligned}
$$




$$
\begin{aligned}
& -\sum_{a_{s+1}} n_{a_{s+1}} \int \mathrm{d} \mathbf{r}_{s+1}\left(G_{a_{1} \ldots a_{s}, a_{s+1}}^{(1)} \hat{H}_{a_{1} \ldots a_{s}, a_{s+1}}-\sum_{i=1}^{s} G_{a_{i} a_{s+1}}^{(1)} h_{a_{i} a_{s+1}}\right) \\
& +\frac{1}{2} \sum_{a_{s+1}} \sum_{a_{s+2}} n_{a_{s+1}} n_{a_{s+2}} \int \mathrm{d} \mathbf{r}_{s+1} \mathrm{~d} \mathbf{r}_{s+2} h_{a_{s+1} a_{s+2}} \\
& \quad \times\left(\hat{H}_{a_{1} \ldots a_{s}, a_{s+1}} \hat{H}_{a_{1} \ldots a_{s}, a_{s+2}}-\sum_{i=1}^{s} h_{a_{i} a_{s+1}} h_{a_{i} a_{s+2}}\right) .
\end{aligned}
$$

The manner of reduction to these equations from equations (41) and (47) demonstrates that the effect of higher potential components on the distribution functions is not simply additive despite the structure of the direct potential, equation (1). Similar calculations using only pair potentials but with a slightly modified ansatz for $f_{a_{1} \ldots a_{s}}$ have been performed by Guernsey [11] and by Ebeling et al. [14]. Last not least, equations (49) and (50) coincide for $s=2$ with the diagrams for the coefficients $\hat{C}_{a b}^{(1)}$ and $\hat{C}_{a b}^{(2)}$ of the binary distribution $f_{a b}$ in the pioneering works by Meeron, equation (5.4) in [15], and by Yukhnovskii and his students [16-19], equation (2.64) in [20], way back to the 1950s. More recently, Holovko and Krienke [21] presented $f_{a b}$ in a purely exponential form, their equations (21), (18), and (22), which after expansion also gets equal to our results.

The detailed knowledge of the distribution function gained in this article constitutes a firm basis for calculating thermodynamic properties. In accordance with the outline sketched in [22], a subsequent paper will be devoted to the derivation of the cluster expansion of the Helmholtz free energy up to the fourth cluster integral [12].

\section{Acknowledgements}

I would like to express my sincere gratitude to the people from the Institute for Condensed Matter Physics of the National Academy of Sciences of Ukraine in Lviv for their warm hospitality. In particular, I am thankful to Ihor M. Mryglod, Myroslav F. Holovko, Vasyl V. Ignatyuk, and Oleg V. Velychko. During the Workshop on Modern Problems of Soft Matter Theory in Lviv, Ukraine, on August 27-31, 2000, I enjoyed inspiring discussions with Ihor R. Yukhnovskii on the binary distribution function and the second virial coefficient for classical Coulomb systems.

\section{References}

1. Bogoliubov N.N. Problems of a Dynamical Theory in Statistical Physics. Moscow, Gostekhisdat, 1946 (in Russian); Translation by E.K. Gora (Geophysical Research Papers, No. 70). Bedford, Massachusetts, Geophysics Research Directorate, 1960.

2. Schmitz G. Zur statistischen Thermodynamik von Systemen geladener Teilchen mit kurz- und weitreichenden Wechselwirkungen. Inaugural-Dissertation. Rostock, Universität Rostock, 1967 (unpublished, in German).

3. Schmitz G. // Ann. Physik (Leipzig), 1968, vol. 21, p. 31.

4. Morita T. // Progr. Theor. Phys. (Japan), 1959, vol. 22, p. 757. 
5. Ebeling W. // Ann. Physik (Leipzig), 1968, vol. 21, p. 315; ibid., 1968, vol. 22, p. 33; ibid., 1969, vol. 22, pp. 383, 392; Physica, 1968, vol. 38, p. 378; ibid., 1968, vol. 40, p. 290.

6. Falkenhagen H., Ebeling W., Hertz H.G. Theorie der Elektrolyte. Leipzig, Hirzel, 1971 (in German).

7. Kahlbaum T. // Contrib. Plasma Phys., 1999, vol. 39, p. 181.

8. Kahn B., Uhlenbeck G.E. // Physica, 1938, vol. 5, p. 399.

9. Friedman H.L. Ionic Solution Theory - Based on Cluster Expansion Methods (Monographs in Statistical Physics and Thermodynamics, Editor: I. Prigogine, Volume 3). New York · London, Interscience Publishers, 1962, Sections 12 and 14.

10. Kelbg G. Einige Methoden der statistischen Thermodynamik hochionisierter Plasmen. - In: Ergebnisse der Plasmaphysik und der Gaselektronik, Band 3. Herausgeber: R. Rompe und M. Steenbeck. Berlin, Akademie-Verlag, 1972, p. 32-34 (in German).

11. Guernsey R.L. // Phys. Fluids, 1964, vol. 7, p. 792.

12. Kahlbaum T. // J. Phys. IV (France), 2000, vol. 10, p. Pr5-455.

13. Schmitz G. // Phys. Lett., 1966, vol. 21, p. 174.

14. Ebeling W., Kelbg G., Schmitz G. // Ann. Physik (Leipzig), 1966, vol. 18, p. 29.

15. Meeron E. // J. Chem. Phys., 1958, vol. 28, p. 630.

16. Yukhnovskii I.R. // Ukrainian J. Phys., 1959, vol. 4, p. 167 (in Ukrainian).

17. Yukhnovskii I.R. // Doklady Akademii Nauk SSSR, 1959, vol. 126, p. 557 (in Russian); English Translation: Soviet Physics - Doklady, 1959, vol. 4, p. 617.

18. Yukhnovskii I.R. // Adv. Plasma Phys., 1974, vol. 5, p. 51.

19. Yukhnovskii I.R., Vysochanskii V.S., Holovko M.F. // Ukrainian J. Phys., 1975, vol. 20, p. 817 (in Russian).

20. Yukhnovskii I.R., Holovko M.F. Statistical Theory of Classical Equilibrium Systems. Kiev, Naukova Dumka, 1980, p. 76 (in Russian).

21. Holovko M.F., Krienke H. // Mol. Phys., 1989, vol. 68, p. 967; note that the fifth diagram in their equation (22) lacks a thick diagonal line as it appears in the sixth one.

22. Kahlbaum T. Statistische Thermodynamik nichtidealer Quantenplasmen. - In: Innovative Forschung in der WIP-Nachfolge - Leistungen und Perspektiven. Herausgeber: G. Seifert und J. Sobottka. Berlin, Humboldt-Universität zu Berlin, 2000, p. 43 (in German). 


\section{Функції розподілу для заряджених частинок з багаточастинковими взаємодіями}

\section{Т.Кахельбаум}

Інститут плазми і лазерної техніки

D-15745 Уілдау, Німеччина

Отримано 14 серпня 2000 р.

Ми розглядаємо багатокомпонентну систему заряджених частинок, які взаємодіють через загальний багаточастинковий потенціал, і обчислюємо функції розподілу з ієрархії рівнянь Боголюбова-БорнаҐріна-Кірквуда-Івона у формі екранованого віріального розкладу до другого порядку включно.

Ключові слова: заряджені частинки, функція розподілу, ієрархія ББГКІ, віріальний розклад

PACS: 05.20.-y 